\title{
THE MABE MIDDLEWARE
}

\author{
Alois Reitbauer ${ }^{1}$, Alessandro Battino ${ }^{2}$, Bart Saint Germain ${ }^{3}$, Anthony \\ Karageorgos ${ }^{4}$, Nikolay Mehandjiev ${ }^{5}$, Paul Valckenaers ${ }^{3}$, \\ 'Profactor Produktionsforschungs GesmbH, Wehrgrabengasse 1-5, A-4400 Steyr, AUSTRIA \\ alois.reitbauer@profactor.at \\ ${ }^{2}$ Institute of Production Engineering and Machine Tools (IFW) - University of Hannover, \\ Schlosswender Strasse 5,30159 Hannover, GERMANY \\ battino@ifw.uni.hannover.de \\ ${ }^{3}$ K.U.Leuven-P.M.A. Celestijnenlaan 300B,B-3001 Leuven, BELGIUM \\ \{paul.valckenaers,bart.saintGermain\}@kuleuven.ac.be \\ ${ }^{4}$ Dept. of Communications and Computer Engineering, Univ. of Thessaly, 37 Glavani - 28th \\ OctoberStr, 38221 Volos-GREECE, karageorgos@acm.org \\ ${ }^{5}$ Dept. of Computation, UMIST, Sackville Str. M60 IQD, Manchester, UK \\ mehandjiev@acm.org
}

\begin{abstract}
The research project MaBE aims at the development of agent-based middleware supporting cooperation in open business environments, based on real-world case studies concerning virtual enterprises. These case studies provide us with a library of scenarios of inter-and intra-organisational cooperation, which impose certain requirements to the construction of effective agent-based middleware platforms supporting such cooperation. However, existing FIPA compliant, agent construction frameworks are insufficient to implement such agent-based middleware, as they cannot meet certain requirements imposed by the case studies. For example, the requirements involving dynamic handling of multiple and evolving ontologies, security and trust issues as well coordination in open environments cannot be directly addressed by current agent development tools and further research is required before building the appropriate middleware functionality supporting them. Since these issues are perceived as key enablers for open business collaboration, this paper provides an overview of the research undertaken to address them.
\end{abstract}

\section{INTRODUCTION}

The V irtual E nterprise (VE) paradigm (Cam, 2001) describes the development of mechanisms to support collaboration of existing business entities in a distributed environment. Multi-agent systems (MAS) are considered to be a suitable approach for modelling various cooperation scenarios within a Virtual Enterprise. Existing agent platforms like for example JADE (Jade, 2004), however, lack built-in support for some important requirements stemming from these scenarios. 
The EU funded research project MaBE is developing a middleware system based upon a FIPA compliant agent platform (FIPA, 2002a) for supporting the development of collaborative business environments. MaBE focuses on extended and virtual enterprises and the applications prototyped within the project reside in the sector of productive industry and logistics Within this remit MaBE has focused on several important areas where further research is necessary to provide effective agent-based support to a number of real-world collaboration scenarios within Virtual Enterprises.

This paper provides an overview on the main research issues addressed. Because of the apparent diversity of research issues, the aim is to provide a holistic broadbrush picture of the set of issues rather than to go into a considerable level of depth exploring an individual issue.

The rest of the paper is organised as follows. In the next section we will present an exemplary industrial use case showing the requirements needed for collaboration within an Extended and Virtual Enterprise (Cam, 2001). Section 3 presents a reference model describing different forms of collaboration depending on organizational structures. Based on this reference model a set of required extensions to current multi-agent systems is described. Section 4 presents Coordination mechanisms for Supply Networks followed by a discussion of trust and security aspects in Section 5. Section 6 provides an overview of issues regarding communication semantics that have to be addressed for enabling cooperation.

\section{INDUSTRIAL USE CASE}

The solution described below is motivated by requirements from real world industrial use cases. As an example, the scenario of a company performing hardening processes is presented. The company consists of nine SME-organized as a Virtual Enterprise where new companies are dynamically joining and leaving the group and new processes are continuously introduced as needed.

The main objective for forming a Virtual Enterprise was the optimized usage of information as well as physical resources. However this possibility cannot be fully exploited due to the amount and the complexity of the information stored (e.g. parttreatment programs, quality control data, production plans). In the following the main impediments for collaboration within one company as well across companies are presented.

Within a single company, the main concerns are related with the production coordination. At present, the definition of batches and the scheduling are activities carried out manually and therefore implying big efforts. In order to reach a higher level of flexibility, there is the need for a resource coordination system allowing production planning, which takes into account the actual workload of the facilities, as well as real-time data about breakdowns and other disturbances.

Interaction between the companies induces mechanisms for communication (flow of information) as well as logistics (flow of material). These mechanisms make use of services currently available within the virtual enterprise but additional services not yet available are needed. Choosing the structure of a Virtual Enterprise it is expected to enable all involved entities to act as if they belonged to just one company, exploiting at the same time the logistic advantage constituted by a 
distribution of the factories in the territory. The transport of the parts (logistic service) can be carried out with VE means, with means of the customer or through logistic providers. The parts received from one customer can have as a destination the same customer of origin or another customer or even another company of the Virtual Enterprise (in case of distributed processes). The resulting number of possible interactions can only be efficiently coordinated with a sy stem supporting automatic selection of services. Since such a system would connect different actors of the supply chain, topics like trust and security have to be addressed. It is, in fact, fundamental to provide a sufficient visibility in order to permit a services discovery, but without revealing reserved information or compromising the security of the communication. Moreover, to allow the interoperability between companies working in different business fields, a common language or at least a common frame of reference is required. For instance the data about a transport can be described in different ways inside the Logistic Provider and inside the Virtual Enterprise; and new concepts can be used when a new transport unit is introduced.

\section{REFERENCE MODEL FOR COOPERATION}

To provide an overall framework for analysing and understanding the case study, for modelling and implementing cooperation scenarios and for handling the requirements implied there, it is necessary to employ a theoretical reference model of collaboration within a Virtual Enterprise. The model on Figure 1, based on (Cam, 2001), was found to fit the scenario described above, and to resonate well with authors' experience. The model is based on different types of collaboration as follows.

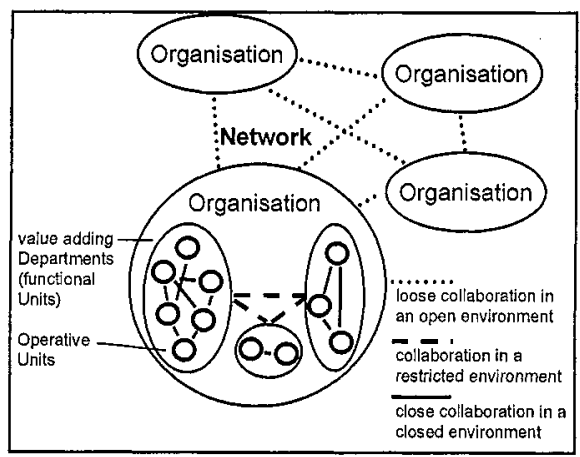

Figure 1 - Levels of Collaboration

Organisations at the lowest level are built on operative units representing atomic resources. These units cooperate among each other forming functional units also called departments or groups. Interactions between these functional units represent the collaboration taking place at enterprise or intra-organisational level. Networks of organisations are the next level of collaboration. Inter-organisational cooperation can further be divided into cooperation of a fixed number of partners or cooperation in an open environment where partners permanently can appear and disappear. Figure 1 presents a graphical representation of the different level of collaboration. 


\section{SUPPLY NETWORK COORDINATION}

As presented in the use case above coordination not only implies coordination of all orders and resources meaning production logistics but also information logistics. A satisfactory solution has to provide monitoring and short term forecasting abilities as well as decision taking mechanisms. While Section 2 presented a static view of various forms of collaboration, a dynamic view on the system is required for resource coordination. The Supply Network paradigm (SAI, 2004) is seen as fitting well in here.

An entity in a supply network is defined by the services it provides to the environment. Combination of entities can either be established by establishing relations or by aggregation. An important factor concerning the established structure especially in open collaboration environments is that it is by no means fixed. The structure will dynamically change while the environment is changing.

Both in intra- and inter-organizational collaboration, autonomy and specificity of an entity has to be respected. As shown in Section 3 there is no single level of autonomy and specificity evolved in the collaboration scenarios. While in intraorganisational collaboration there is only single goal to be achieved among participating entities this is not the case in inter-organisational collaboration. Production entities in the sy stem will be striving towards different and often a lso conflicting goal. Still, decisions made by one entity have to be respected by the other entities. Consequently a coordination mechanism must be able to define win-win solutions for all involved entities.

For the definition of a supply chain architecture the PROSA (HAD, 2003) model has been chosen. PROSA allows the modeling of holonic system structures using three main building blocks called holons.

- Product holons p rovide the p rocess knowledge of a product, meaning a 11 possible production steps combination and their order.

- Order holons manage one order (customer order, production order, a aggregation of orders, etc) throughout all production stages.

- Resource holons represent a material or immaterial resource within a supply network.

In addition to the architectural building blocks a mechanism for coordination of these entities is required. Open environments impose due to their dynamically changing structure great complexity regarding coordination. Centralised approaches are $n$ ot a pplicable, a s they r equire the perception of the whole e nvironment. $T$ his however is either not or almost impossible in open environments.

The coordination mechanism presented here is based on the insights from the behaviour of ants searching for food. The main advantage of such an ant-based design is that individuals a re not e xposed to the complexity and dynamics of the global situation.

There are three different types of ants and respectively three stages of the resource coordination process each one fulfilling one dedicated purpose.

Feasibility ants are responsible for discover the topology of the supply network; finding out all respective services there can be accessed. This information can be used by explorer ants, which are responsible for exploring possible solutions through the supply network for a specific order. After the selection of a possible candidate solution found by one of the explorers, intention ants are used to spread the order 
agent's current intention along all resources involved in the orders respective objective. Based on these declared intentions, service providers can build a dynamic load forecast, which can be used by explorer ants to make their performance estimate reliable.

All information produced during each of the three phases has only a limited period for which it is valid. After this period, the information vanishes if it is not produced again. This procedure represents an analogy to the evaporation of pheromones - the analogy of information - used in ant communication. As a consequence of this evaporation of information each of the phases above has to be executed cyclically. This approach keeps information in the system always up-todate and additionally and even more important makes the system robust to changes.

\section{TRUST AND SECURITY}

Current agent systems lack proper support for security and other trust building mechanisms (Pos, 2000). A trusted environment is however a vital point in business collaborations. Current standardisation activities within FIPA (FIPA, 2004) deal with the implementation of basic security mechanisms for message based security. Security mechanisms however are only one factor of trust. Reputation and confidence building mechanisms are additional factors that have to be addressed. As not all of these factors are relevant for any kind of collaboration - a clarification on this topic is needed.

Requirements for trust issues depend on the form of collaboration. We will now relate them to the model presented in Section 3, concentrating on where ICT support is needed.

Collaboration within departments happens within a closed system and in a trusted environment. An established legal framework forms the basis for confidence in the actions of the parties involved in collaborations. This confidence is supported by striving towards a common goal and by the fact that the acting parties are employees of the same company, bound by contracts.

Inter-department collaboration happens on a regular basis but less frequent than within a department. The collaboration scenarios are well defined and all actions take place in a closed, trusted environment as well. Trust however declines due to reduced personal contact in collaborations. Security at this level addresses the protection of information and resources based on organisational roles as well as on the context of collaboration.

In inter-organisational collaboration the environment is insecure, more dynamic, and in general not trusted. Security for collaboration scenarios within an organisation is completely controlled by the organisation itself. In an open environment a part of this control is handed over to the other involved parties. Mechanisms are needed which allow communication scenarios, in which not all parts of the infrastructure are controlled by the organisation itself.

Contracts between companies are as powerful as contracts regulating interorganisational activities. However they more difficult to enforce as independent third parties are required for enforcement. As companies might have conflicting or competing goals, confidence in the actions of others is not present per se. The 
establishment of reputation also takes longer as interactions occur on an infrequent, business need driven basis.

In inter-organisational collaboration different scenarios of doing business exist. Collaboration based on strictly defined processes with known business partners represents the simplest form of inter-organisational collaboration. This can be extended to a fully open dynamic organisational network, where processes are not fixed, and collaboration takes place with unknown partners.

A different $v$ iew on the roles of the importance of trust related issues a re the states collaboration goes through in its lifecycle. Following (Cam, 2001) collaboration has four states. The initial state is the creation state, followed by the operation of the virtual organisation. During operation the organisation is suspect to evolve by changing its structure. The dissolution of the virtual organisation is then the last stage. The evolution stage can however be eliminated in closed and fixed collaborations. Confidentiality and data origin identification play a vital role in all stages. Authorization issues as well as reputation and confidence enforcing mechanisms are key requirements for the execution phase. The creation and respectively evolution phases are responsible for setting up and defining reputation and confidence mechanisms.

Due to the s pecific r equirements to a uthorization mechanisms in collaborative environments (Edwards, 1996) that cannot be coped with using standard mechanisms like access control lists (ACLs) or access control matrixes (ACMs) as described in (Lampson, 1974). Although there is a lot of work available on reputation and $c$ onfidence $b$ uilding mechanisms, there is no ready to use solution available for business applications. Agent-oriented systems however offer a very attractive way for implementing these features.

By applying the mediator pattern (Gamma, 1994) agent communications can be intercepted and trust related checks can be performed based on interaction between agents. This mechanism is for example also used for systems monitoring the access of medical databases ( $\mathrm{Liu}, 2000)$ or access control in web service environments (Sko, 2003).

\section{ONTOLOGY MANAGEMENT}

To meet the semantic interoperation requirements at the tactical level in the presented case study, it is preferable to use ontologies, which are formal representations of the meaning of a set of concepts and their relationships in a domain of discourse. The use of ontologies is currently a fundamental part of agent behaviour (FIPA 2002) and it is in principle consistent with other approaches such as those used in Semantic Web and Enterprise Application Integration. However, the use of ontologies within the multi-agent systems community is biased towards semantic interoperation, leaving two technical research issues to the developers of business support systems such as MaBE: ontology mapping and dynamic ontologies.

\subsection{Mapping of multiple ontologies}

The need for ontology mapping stems from the fact that different views of the same reality may exist between collaborating partners. To achieve common understanding, there are three main approaches: 
- Using current standards, (i.e. XML-based representations) all systems can communicate using the least common denominator of all ontologies. This causes the loss of information and respectively reduces the communication abilities making deep integration impossible.

- Create a common "world model" integrating all sub-models of all involved systems. The model created using this approach is undoubtedly very complex. Additionally this approach cannot be taken in open environments where not all models will be known at design time.

- Finally, mappings on-demand can be created between the ontologies of interested parties. This would keep the size of the ontologies low and minimise information loss. The mapping process would be distributed throughout the collaborative environment.

We have taken the Partially Shared Views approach (Lee, 1990) to collaborative ontology mapping on-demand, and created appropriate agent negotiation mechanisms implementing this within MaBE.

\subsection{Dynamic handling of evolving ontologies}

Interoperation at tactical level of the case study requires that ontologies may change at run time. The middleware platform should therefore provide facilities for handling evolving ontological concepts at run-time using one the following approaches:

a. Using an external Ontology Server is a promising approach to realising dynamic ontology management. The server acts as a run-time middleware, which administers the knowledge base containing various ontologies. It includes powerful $r$ easoning mechanisms (inference e ngines) which deliver a nswers to knowledge base queries submitted by the client applications. Applications using the ontology server are easily adaptable to new data and information by changing the underlying ontologies and knowledge bases.

b. Using Ontology Agents as mediators is the preferred mode of implementing an Ontology Server in a gent-based systems s ince numerous complex interactions with agents will be required. The Ontology Agents will provide translation, mapping and interoperation services, and act as mediator to agent interactions involving unknown ontological elements. They will also be responsible for storing and manipulating ontology evolution on run-time. So other agents will not be concerned with ontology management, but focused on the usage of ontologies to provide application functionality.

c. Providing direct access to evolving ontologies to each agent. Although appealing, the approach of having ontology agents being responsible for all ontology management operations is likely to result to communication explosion and to inefficient infrastructure. Therefore, our view is that Ontology agents should be responsible for storing and manipulating evolving ontologies but once any changes or conflicts have been resolved, for example through negotiation of ontological concepts, then individual agents should be able to use the updated ontology versions. This would require appropriate interaction protocols for ontology management and versioning.

Each of these approaches is more suitable for a particular style of collaboration from Section 3, for example the final one is tuned to the needs of intra-departmental collaboration whilst the first one is appropriate at inter-organisational level. The 
MaBE middleware will thus have to provide an integrated approach to dynamic ontologies based on a combination of these approaches. An initial draft of this approach has been published elsewhere (Carp, 2004). Experiments are currently under way to determine the optimum system configuration with respect to the optimal integration of the three approaches.

\section{CONCLUSION}

The MaBE middleware aims satisfy a set of currently unfulfilled requirements for supporting business collaboration identified from a business collaboration reference model and representative case studies: the requirements of coordination, trust and security, and ontology management. In this paper the approaches followed to meet the above challenges are outlined: Coordination is based on a simple and powerful modelling framework - PROSA - which is combined with nature inspired coordination mechanisms designed for dynamic environments. Trust and security is based on mechanisms specifically designed for the characteristics of multi-agent systems supporting business applications. Finally, an integral approach to ontology mapping and dynamic ontology handling of evolving ontologies will be extending the ontology mechanisms of the underlying JADE kernel to ensure semantic interoperation of involved parties at all levels of collaboration.

ACKNOWLDEGEMENTS. The work was partially funded by the European Commission under the GROWTH project MaBE (Multi-agent Business Environment), project no. GRD1-2001-40496.

\section{REFERENCES}

1. Camarinha-Matos, L., Afsarmanesh, H.: Virtual Enterprise Modeling and Support Infrastructures: Applying Multi-agent System Approaches. Lecture Notes in Artificial Intelligence, Vol. 2086. Springer Verlag Berlin Heidelberg (2001) 335 -364.

2. Carpenter M., Gledson, A. and Mehandjiev, N., Support for Dynamic Ontologies in Open Business Systems. To appear in Proceedings of the AAMAS'04 AOIS Workshop. 2004.

3. Poslad S and Calisti M. Towards improved trust and security in FIPA agent platforms. Autonomous Agents 2000 Workshop on Deception, fraud, and trust in agent societies, Barcelona, June 2000.

4. FIPA: FIPA Abstract Architecture Specification, SC00001

5. FIPA: FIPA Ontology Service Specification, XC0086.

6. FIPA: FIPA Agent Message Security Object Proposal: FIPA Security TC Working Document (2004).

7. Edwards, W.: Policies and Roles in Collaborative Applications. ACM Conference on Computer Supported Cooperative Work (CSCW'96), Cambridge, Mass., (1996) 11-20.

8. Gamma E., Helm R., Johnson R., Vlissides J.: Design Patterns - Elements of Reusable Object-Oriented Code. Addison-Wesley Professional Computing Series. (1996).

9. JADE: http://jade.tilab.com; Telecom Italia Labs.

10. Lampson, B.: Protection. ACM Operating Systems Rev. 8, 1 (1974), 18-24.

11. Jintae Lee and Thomas W. Malone. Partially shared views: A scheme for communicating among groups that use different type hierarchies. ACM Transactions on IS, 8(1) 1990.

12. Liu D., Law K. Wiederhold G.: CHAOS: And Active Security Mediation System. Proceedings of the 12th International Conference on Advanced Information Systems Engineering. Lecture Notes in Computer Science, Springer-Verlag: London, UK (2000) $232-246$.

13. Skogsrund H. Benatallah B. Casati F.: Model-Driven Trust Negotiation for Web Services, IEEE Internet Computing - Vol (7) No (6) (2003) $45-52$

14. Hadeli, Valckenaers, Zamrescu, Van Brussel, Saint Germain., Holvoet, Steegmans:. Self-organising in multi agent coordination and control using stigmergy. ESOA. (2003).

15. Saint Germain, B., Valckenaers, P., Verstraet, P., Bochmann, O., Van Brussel, H.: Supply network control, an engineering perspective. Accepted at 11 th IFAC Symposium on Information Control Problems in Manufacturing, Salvador, Brasil, (2004). 